\title{
Displaying Heart Rate Data on a Bicycle Helmet to Support Social Exertion Experiences
}

\author{
Wouter Walmink, Danielle Wilde, Florian 'Floyd' Mueller \\ Exertion Games Lab \\ RMIT University \\ Melbourne, Australia \\ \{wouter, danielle, floyd\}@exertiongameslab.org
}

\begin{abstract}
People often engage in physical activity with others, yet wearable technologies like heart rate monitors typically focus on individual usage. In response, we discuss the potential of heart rate displays in a social context, by means of an augmented cycling helmet that displays heart rate data. We studied how pairs of cyclists engaged with this setup and found that access to another person's heart rate data can result in social interplay which in turn supports engagement with the exertion activity. Through our design process and study, we reveal key dimensions of designing for social uses of heart rate data and wearable displays: temporal and spatial accessibility of data, technology support for its interpretation, and influences on heart rate. We also articulate a set of insights for designers that aim to support social exertion activities with heart rate data. As such, our work expands our understanding of wearable technologies' unique interaction opportunities.
\end{abstract}

\section{Author Keywords}

Heart rate; biofeedback; body-worn technologies; wearables; exertion; cycling; helmet.

\section{ACM Classification Keywords}

H.5.2 [Information Interfaces and Presentation]: User Interfaces - Interaction styles.

\section{INTRODUCTION}

Digital technology is increasingly used to support physical activities. Examples include GPS-powered mobile phone apps that track distances for joggers and cyclists, watches that measure skin temperature and heart rate monitors that inform sportspeople of their physiological response to exercise. We focus on heart rate monitors, noting that they are typically designed for individual use. A common way to display heart rate data is via a wristwatch [11], making the

\footnotetext{
Permission to make digital or hard copies of all or part of this work for personal or classroom use is granted without fee provided that copies are not made or distributed for profit or commercial advantage and that copies bear this notice and the full citation on the first page. Copyrights for components of this work owned by others than the author(s) must be honored. Abstracting with credit is permitted. To copy otherwise, or republish, to post on servers or to redistribute to lists, requires prior specific permission and/or a fee. Request permissions from Permissions@acm.org.

TEI'14, February 16 - 19 2014, Munich, Germany

Copyright is held by the owner/author(s). Publication rights licensed to

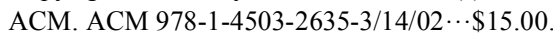

http://dx.doi.org/10.1145/2540930.2540970
}

data easily accessible for the wearer, yet not readily visible to others. This way of displaying heart rate data may work well for a person exercising alone, yet it precludes shared access to the data in a social exertion experience (a digitally supported experience where multiple users engage together as they invest physical effort $[13,14]$, sometimes referred to as Computer-Supported Cooperative Sports [28]).

Our study focuses on non-professional cyclists who ride regularly for fitness and fun, and find cycling with others enjoyable and motivating $[5,10]$. We note that cycling affects heart rate significantly and many cyclists - including social cyclists - use heart rate sensors for self-monitoring. Yet the use of heart rate monitors in cycling has mostly been studied from an individual training-support [4] rather than a social perspective [1]. We therefore consider social cycling to be a good starting point for an exploration into heart rate data use within a social exertion context.

The motivation for our work is three-fold. First, rapidly increasing mobile computing power combined with increased affordability allows for novel experiences around sensor data. iPhone apps such as Zombies, Run! ${ }^{1}$ and Mobile Adventure Walks ${ }^{2}$ measure how far user walk or jog and turn these activities into treasure hunts. Our aim is to deepen our understanding of how existing exertion activities might be reframed into novel experiences, leveraging mobile sensor technologies. Second, mobility is often integral to exertion activities, yet displaying data for mobile users can be challenging. Augmented clothing and body-worn accessories are viable options for display, yet the challenges they present are varied. Sports watches may be used to display a wealth of information, yet the small interface often requires cumbersome user interactions to access different modes. Clothing such as basketball uniforms can be embedded with displays [21], yet present issues such as durability, hardiness, washability and occlusion by layers of clothing. Bicycle helmets do not suffer from many of these constraints. We therefore see the helmet as an underexplored opportunity for technological augmentation. Finally, using helmets to display information allows for an intriguing interaction setup. A helmetmounted display affords access to data for people close-by, but not to the wearer. This situation is in contrast with

\footnotetext{
${ }^{1} \mathrm{http}: / /$ www.zombiesrungame.com

${ }^{2}$ http://www.mobileadventurewalks.com
} 
mobile phone exertion-support systems such as $\mathrm{Nike}+{ }^{3}$, which offer people far away access to exertion data through Facebook and Twitter, but no easy access to those in spatial proximity. We therefore see the limited access to heart rate data as a unique design opportunity.

Our research makes the following contributions: We extend knowledge about computer-supported cooperative sports by providing insights into how digital technology can support social exertion experiences, in our case: social cycling. We articulate how designers may engage heart rate data to support social exertion experiences, and demonstrate the feasibility of using helmets as interactive wearables. Lastly, we contribute to an understanding of the design of interactions where the sensor wearer has less access to sensor data than others nearby. With this research, we hope to guide designers who aim to support physical activity, ultimately helping users profit from the benefits of exertion.

\section{RELATED WORK}

Our research is informed by social aspects of exertion experiences, the use of heart rate data in $\mathrm{HCI}$, and interactive systems for cycling.

\section{Social Aspects of Exertion Experiences}

Social facilitation theory suggests that exercising with others can contribute positively to the experience [26]. In complement, exertion can facilitate social relationships, including when digitally augmented [15-17]. These findings drive research and product development into how technology may be used to make exercise data more socially relevant, though many systems focus on data other than heart rate. IFit $^{4}$ creates the illusion of running with others while on a treadmill, by comparing time and distance data to previously recorded results from others. Garmin Connect ${ }^{5}$ allows comparing achievements with fellow sportspeople online after the exertion activity. The Endomondo app ${ }^{6}$ allows sharing that one is running or cycling, so friends can send text message encouragements that are read out loud. These systems focus on exertion outcomes that can be shared, over distance or time, often after or from outside the exercise activity. In contrast, our research explores shared use of heart rate data during exercise with others who are engaged in the same activity.

\section{Heart Rate Data in $\mathrm{HCl}$}

The use of heart rate data is gaining increased attention in $\mathrm{HCI}$, in particular in relation to computer games. Examples include games that dynamically respond to a player's emotions, as interpreted through heart rate [2, 7] and exertion games that use heart rate as direct input control for a player [18, 19]. Such applications typically focus on single-player experiences. Notably, it has been suggested that social experiences can be augmented when heart rate is

\footnotetext{
${ }^{3}$ http://nikeplus.nike.com/plus/

${ }^{4}$ http://www.ifit.com/

${ }^{5} \mathrm{http}: / /$ connect.garmin.com/

${ }^{6}$ http://www.endomondo.com/
}

used to balance activities between gamers [25] and also joggers [17]. In such cases the data is used to help compensate for differences in physical abilities, in particular when participants share the activity from a distance. We build on these findings by making heart rate data available to co-located cyclists, who are engaged together in the social exertion activity.

\section{Interactive Systems in Cycling}

A study on mobile experiences for riders points to the unique design challenges posed by the distinct nature of cycling [22]. For example, designers must be sensitive to cycling being a demanding activity that may limit attention to a system and social interaction. In regards to safety, Jones et al.'s Smart Helmet [9] can warn the wearer of hazards on the route, while Mind Rider explores how helmets may warn car drivers if the cyclist is stressed ${ }^{7}$. Both these designs focus on interaction between the wearer, the helmet and the surrounding, rather than the social experience of cycling.

Westerink et al. [27] tested how a virtual coach, providing instructions based on the user's heart rate, could affect user experience. Their results confirm that heart rate data can be used successfully as input to digital systems and shared with others (including where the "other" might be a virtual character) [3]. However, the study did not investigate the influence of the system's design on the social experience.

Our review highlights a gap in understanding how social exertion experiences may be supported with digital technology, in particular in a cycling context. To investigate this gap, we present "Open Heart Helmet", a prototypal augmented helmet to explore the use of heart rate data displays in a social cycling context.

\section{EXPERIENCING THE OPEN HEART HELMET}

Open Heart Helmet (fig. 1) consists of a helmet-mountable display (a smartphone) and a heart rate monitor (Zephyr BioHarness $\mathrm{BT}^{8}$ ). We used the open-source Processing platform to communicate with the heart rate monitor and output the data onto the display (fig. 2). On startup, the app establishes a Bluetooth connection and receives a continuous stream of data packages at $1 \mathrm{~Hz}$. On receiving a package, the displayed heart rate is instantly updated.

\section{Participants}

We undertook a study with twelve cyclists. Participants were recruited through the newsletter of an amateur cycling organization, asked to contact us in pairs; 5 male and 7 female active cyclists volunteered. All pairs were familiar with riding together and five of the six pairs were also in a relationship, the other pair were friends. The average age was 47 years, ranging from 25 to 65 . When asked how often they cycle, the answer was on average 4.5 rides per week, with all participants saying they ride at least twice a

\footnotetext{
${ }^{7} \mathrm{http} / /$ web.media.mit.edu/ arlduc/projects/mindrider/

${ }^{8} \mathrm{http}: / /$ www.zephyranywhere.com/products/bioharness-3/
} 


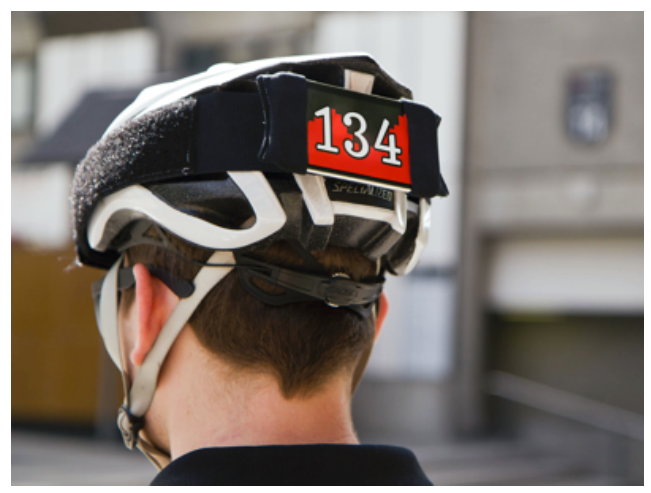

Figure 1. Displaying the cyclist's heart rate on a helmet.

week. All cycle to benefit their fitness, most also cycle for fun or leisure, and five had participated in cycling competitions, though none professionally. During the study, the paired participants met at different times in a public park commonly used by cyclists. Australian law requires cyclists to wear a helmet, meaning all participants brought their own helmet. Participants also used their own bikes. Due to legislation as well as ethics and safety aspects concerning a prototypal helmet design, we told participants to not go beyond the park onto roads with car traffic. We focused on the immediate ride experience rather than longterm use of an augmented helmet, with each session lasting around 1.5 hours. The participants shared the path with other cyclists, joggers and people walking their dogs.

\section{The Rides}

We began by ensuring that the augmented helmets were sitting comfortably and adjusted correctly. From our pilot study we learned that it could be beneficial to guide the focus of the study on the heart rate interaction. We therefore started all sessions with the suggestion to aim to reach a specific target heart rate. Participants could select their preferred target heart rate based on previous usage of heart rate monitors (if they had used heart rate monitors before), or use the heart rate monitor provided to determine a suitable target that was challenging but not overly demanding. Although we started off with suggesting this basic "game", we did not instruct the participants how to reach the target heart rate. We also did not make any further suggestions how they should interact with the Open Heart Helmet, as we were interested in the strategies they would employ to interact with the system and each other.

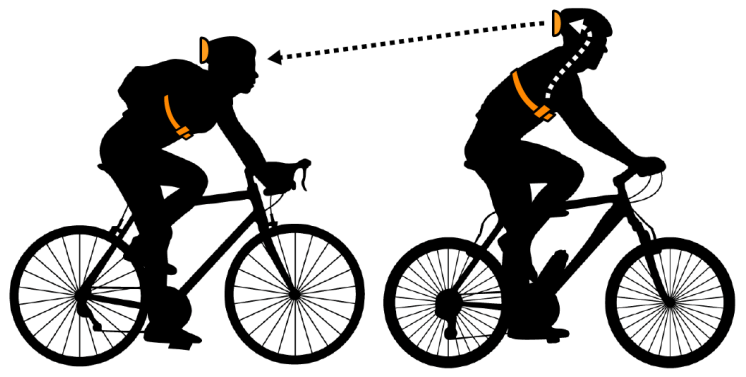

Figure 2. The sensor on the chest sends heart rate data to a display on the helmet, viewed by the other cyclist.
The primary information displayed was heart rate, represented as large, bright numbers. Secondary information also displayed included a graph visualizing the progress of the heart rate over the last minute of the ride behind the large number, or alternately, a visualization indicating how far participants were from their target heart rate. While participants experienced both secondary visualizations, in the interviews none reported on having used them during the rides. We therefore exclude them from our discussion.

After the cycling activity we conducted a semi-structured interview with each couple, which lasted around half an hour. All interviews were audio-recorded and transcribed by the first author. We then identified dominant themes through an iterative reading and grouping process. We checked these themes against the data and tried to articulate them in plain language, facilitating our thinking through writing. We then categorized these themes into three sections that form the basis of the following findings.

\section{FINDINGS}

We examined the participants' responses to the system, and the strategies they used to interact with the system and each other. In the following discussion, we refer to the person whose heart rate was talked about as the "actor" and the other person as the "observer". While we make a distinction here between the observer and the actor, the participants took on both roles, depending on whose heart rate they talked about. We link each participant's quote back to the session it was expressed in: e.g. "S1" means session one.

\section{Cyclists' Experiences}

Dependence on a Partner: A theme brought up by participants during the interviews was the experience of depending on someone else for heart rate readings. Five participants expressed a desire for direct access:

"Yeah, I was desperate to know, I wanted to pull my helmet off and have a look. [...] It felt like I was blind." (S1)

However, a sense of appreciation of the dependence also emerged:

"It is fun when you have no idea what you are supposed to do and you totally rely on someone telling you to slow down." (S4)

It appeared that the participants who predominantly cycle for leisure were more open to the idea of dependence, while the more performance-oriented participants, in particular those familiar with heart rate monitors, preferred seeing their own heart rate data.

Values of Being an Observer: Participants unanimously expressed enjoyment playing the role of the observer. They thought it was fun and provided a potential for distraction from the discomfort of exercise:

"I was having fun providing the feedback." (S4)

"I enjoyed reading [the data] off the back of [my partner]'s head. It seemed the circuit was [...] going a lot faster." (S1) 
In addition, the heart rate data was identified as a seed for the social interaction:

"I enjoyed whether it was on your head or my head, I enjoyed the interaction part of it, I guess, talking to each other." (S1)

Cyclists pushing each other to go faster may unintentionally promote over-exertion. One participant's comment shows how observing someone else's heart rate data made him more aware of this risk:

"[In past rides] I never felt I was pushing anyone too far, but [using this system made me aware] I have."

[Partner]: "[confirmative:] Yeah!” (S6)

Facilitating a team experience: While cycling in a competitive context is often a team sport, it may be less so during fitness or leisurely bike rides. Five participants reported a sense of shared experience through our system, which facilitated working together as a team.

"I think I really like working as a team. It made it more fun." (S5)

One participant explained how this sense of being a team was caused by the system moving her attention away from herself and towards her partner:

"It made us work together as a team to reach individual goals. Instead of thinking 'what am I doing', I was thinking about what the other person was doing and how can I help [him]." (S6)

Another participant's response was that he felt his partner's challenge to be his own, because his partner depended on him:

"[...] it's an equal challenge, because she doesn't have a clue what's going on, except for the information that I am providing, so it's just as much a challenge for the person behind as it is for the person trying to bring their heart rate down or up." (S4)

We noticed taking on the other person's challenge may not just entail helping them reach their goal, but also taking care of them in the process.

"To get [my partner's] heart rate up was a matter of, looking ahead, making sure there was nothing she was going to crash into." (S6)

Six participants applauded how the system supported them in engaging in a social playful experience:

"The moment the person in front hits the lower limit..." (Partner interrupts:) “'OK, we're off now!' Definitely some fun in it." (S5)

Privacy and safety concerns while cycling: One issue touched on in previous research [6] relates to public exposure of personal data such as heart rate. Those we asked directly about privacy did not suggest it was an issue for them:

'I don't know, who cares. [...] I wouldn't care if they [other cyclists] knew [my heart rate], I wouldn't care if they didn't." (S4)
Six of the participants were wary of the distraction the screen could cause on public roads:

"I noticed when I was reading off the back of [my partner's] head [laugh] I wasn't really paying attention to what I was doing." (SI)

"[I was] a little bit wary, because we were sharing the road with other users, and in the first round we weren't sure what to expect. After that it was fine." (S3)

\section{Observer Tactics}

Observers informed, instructed and motivated the actor in many, sometimes unexpected, ways.

Sharing numbers, interpretations and instructions: All participants informed their cycling partner at some point during the session about the numbers on the display.

"[I said] what speed the heart rate was, like 'you're at 120, you're at 130'." (S4)

In addition, participants interpreted the raw numbers. They translated the heart rate readings into informative messages about changes and progress:

“I was saying 'It's coming down, it's coming down.' [...] Wow, stop! Ah, too late..." (S5)

Another tactic used was to give instructions ranging from simple one-word shouts to more elaborate directives:

“To start off with, I was just saying 'harder' or 'slower'. By the end I was doing it more by the numbers, [...] even counting down, like '5 more, 3 more, 2 more. Green!', (S5)

Personalization and creative ways to affect the actor: Three observers also reported adding subjective reflection to the heart rate information they conveyed. They would praise the other person for doing well, or encourage them to reach the target.

"[I was saying:] 'Looking good, you're nearly there. That's it, beautiful, go again. '" (S4)

The creativity some participants exhibited impressed us. In particular, one observer explained how he tried to lower the actor's heart rate by verbally describing pictures associated with calming sensations, facilitating a new exertion experience of 'meditation cycling':

"And then to lower it down, you know, to 120 or whatever it was, it was just my soothing voice."

"He was telling me to imagine all these calming things."

"A nice breeze, a beautiful field, you know, relaxing. And it really worked." (S6)

Cycling behind the actor, observers mostly used verbal communication. However, one participant discovered that cycling close could prompt the actor to speed up:

"I would chase her and that was enough to get her up to 140." (S5)

\section{Actor Tactics}

When informed about their heart rate through their partner, actors used a range of tactics to respond. Not surprisingly, the default method to raise or lower heart rate was by changing speed. 
"To get my heart rate up, I just had to go really fast." (S6) However, participants expressed frustrations with the fact that a low target encouraged them to peddle less.

"I think the only frustrating thing was [my partner] telling me I had to stop peddling." (S5)

Participants also changed gears to affect their heart rate. However, sometimes they had to slow down for pedestrians and dogs. Such environmental obstacles forced participants to use different tactics to raise their heart rate without cycling faster. Furthermore, one couple needed to compensate for differences between bikes and fitness levels. Apart from changing gears, they orchestrated their situation to allow for a major sprint in low gear:

"I was yelling out his numbers again and kind of sprinting off so he would have to come and catch me in his tiny little gear."

"We were coordinating our movements so she could spot me as I was going passed her at that speed." (S6)

\section{KEY DIMENSIONS OF SHOWING HEART RATE TO OTHERS}

We now articulate a set of key dimensions that describe the design space of systems that display heart rate to others. The dimensions were derived from our analysis of related work, our craft knowledge, including the consideration of design alternatives, gleaned from designing Open Heart Helmet. Insights gained from participants during the study allowed for further refinement. Although these do not cover all design alternatives across the design space, our intention is to present designers with the range of design opportunities available to them in order to guide the development of a diverse set of future systems.

\section{Dimension 1: Temporal Accessibility of Heart Rate Data} This dimension concerns when heart rate data is accessible to others. We identify three key possibilities for designers:

1.1 Throughout the Exertion Activity: Designers can choose to make heart rate data available at all times throughout the exertion activity. However, competing cognitive and physical demands may make access challenging or a safety issue. In Open Heart Helmet the participants had to divide their attention mainly between the display and the cycling path, where sometimes the focus on the path had to take priority (for example when navigating a narrow bridge). In contrast, when making heart rate data available in an indoor context, not uncommon for exertion games [25], such focus shift demands are likely not as prevalent and associated with a lower risk for accidents.

1.2 At Specific Times During the Exertion Activity: Designers can choose to make heart rate data accessible at specific times during the exertion activity. A system could detect a low point during the activity in exertion or cognitive demand and offer heart rate data only at this point in time. In Open Heart Helmet, participants only had access to heart rate data when they were behind the display wearer.
1.3 Before and after the Exertion Activity: Designers can choose to only make heart rate data available before and after the exertion activity. For example, user of Suunto Quest ${ }^{9}$ can analyze their heart rate data after the activity, supporting a reflective process independent of the timepressure and attention-demands of the exertion activity. This approach affords comparisons of heart rate data across different activities, across multiple activity times and across several actors.

Dimension 2: Spatial Accessibility of Heart Rate Data This dimension is concerned with the extent to which display position affects heart rate data access. In exertion activities, the body is often moving through space [14], hence spatiality can be a key aspect for accessibility. We identify two general possibilities for spatial accessibility:

2.1 On the Body: Heart rate data can be displayed on the body. This approach affords an association between the data and the source of the data [21]. For example, wearing the Open Heart Helmet suggests that the heart rate displayed is coming from the wearer (in contrast to being sensed from another person). However, in Open Heart Helmet, the design choice to display heart rate data on the rear of the helmet meant that access by others was limited to people behind the wearer.

2.2 Removed From the Body: Positioning the heart rate display away from the body, for instance on a large public display [20], may make it easy for others such as spectators [24] to access heart rate data. However, this setup can make the ability to associate the data with its source more challenging. Designers who opt for this setup may need to consider ways of associating the display with the participant whose heart rate is represented, for example by using labels to associate data with an individual.

\section{Dimension 3: Support for Interpretation of Heart Rate Data}

Heart rate data provides insight into how a person's body is responding to an exertion activity. However, the raw data may not necessarily be easily understood. This dimension therefore describes the extent to which the system supports observers in interpreting heart rate data.

3.1 Supplementary Information: Supplementing raw heart rate data with information such as a recommended heart rate range, a target heart rate, a person's maximum heart rate, or other relevant data, specific to the participant, context or exertion activity, may enrich the understanding of the data and hence support the emergence of meaningful interpretation.

3.2 Representation of Heart Rate Data: Open Heart Helmet offers heart rate data primarily as a number. Alternative representations may support the interpretation process. For example, changes in data over time made visible using

\footnotetext{
9 http://www.suunto.com/global/en/products/Heart-Rate-
} Monitors/suunto-quest/suunto-quest-black-running-pack 
graphical visualization methods might make interpretation easier. Information about heart rate fluctuations may also support the interpretation of the data as a warning signal.

3.3 Shared Exertion Context: With Open Heart Helmet, cyclists ride together, facing the same incline, perhaps startled by the same dog, etc. Such factors can affect heart rate. As both riders experience these factors together, we call this a shared exertion context. The shared context facilitates the interpretation of heart rate data. This shared exertion context is often only supported to a limited extent in distributed experiences, like when cycling on stationary exercise bikes with friends over the internet with the TacxVR system ${ }^{10}$ : if one rider is startled by their dog at home, other riders may not realize this. Limited shared context hinders the ability for remote riders to interpret sudden changes in heart rate.

\section{Dimension 4: Influences on Heart Rate}

Our last dimension is concerned with various influences on the user's heart rate, which may inspire the system' design.

4.1 Environmental Effects: The environment can affect heart rate, for example a dog startling a rider. Weather and road conditions may also have an effect. Our riders were not given an option to ride in different weather conditions, at different altitudes or over a different route. However, the unpredictable nature of the publicly accessible outdoor environment we used impacted the riders' heart rates.

4.2 Effects initiated by the Sensor Wearer: People can affect their own heart rate in a number of ways. With Open Heart Helmet cyclists can influence their heart rate by cycling faster or slower, but also by changing gears: we remind designers that the exertion equipment also offers opportunities to affect heart rate.

4.3 Effects initiated by Others: Other people can also influence a person's heart rate, for example through verbal encouragement. Having access to heart rate data of another person allows users of Open Heart Helmet to observe directly how their cheering, chasing or other behaviors affect their partner.

\section{DISCUSSION}

Our dimensions articulate design options when displaying heart rate data in a social exertion context such as cycling. Yet these dimensions do not tell designers how their choices might affect the resulting user experience. In response, we now discuss a set of design insights derived from our study in relation to the dimensions. We refer to the dimensions by shorthand notation: "D1" is dimension one. Insight 1: Sharing and Affecting Each Other's Heart
Rate can Facilitate a Social Exertion Experience

Current approaches for accessing heart rate data (e.g. wristwatches and smartphone apps) rarely support colocated cyclists in being involved in each other's exertion

\footnotetext{
${ }^{10} \mathrm{http}: / /$ www.tacxvr.com/
}

activity. If at all, they focus mostly on post-session involvement (D1.3). This is probably because they are targeted at people aiming for performance enhancement. However, LaChausse's work [10] suggests designers should also support cyclists who primarily enjoy the social ride experience. In response, our study illustrates various ways in which making heart rate data socially available could promote a shared exertion experience during a casual cycle (D3.3). First, sharing heart rate data may foster the sense of being a team by turning personal goals into mutually shared goals. Second, being able to see someone else's heart rate can help to empathize with another person, for example by seeing when and why someone may be close to his or her maximum heart rate (D4). Lastly, the system can provide a playful open framework to get involved in each other's heart rate.

Five of our twelve cyclists articulated that access to each other's heart rate supported the perception that they shared an exertion experience and that they were "in it together" (D1.1,D2). They noted this was supported by the ability to affect each other's heart rate (both through words and actions, enabled by the shared context (D3.3)). Cycling may be considered a parallel activity, in which cyclists act independently; by that we mean they are not bodily interfering with each other's exertion activity (unlike in wrestling for example) [15]. With the Open Heart Helmet the cyclists had the opportunity to receive direct feedback on what effects their actions had on their partner's bodily experience. During a traditional bike ride, it might be difficult to discern what the effect is if one yells a motivational chant at a rider. In contrast, the Open Heart Helmet offers the observer immediate feedback through the display on what effects his/her actions may have on the bodily experience of the cyclist (D4.3). In result, the cyclists' perceptions of the extent to which they were able to bodily interfere with each other appeared to be enhanced. This turned the parallel activity into a more interdependent one, which appeared to facilitate togetherness. Our results therefore extend prior heart rate research on jogging (also a parallel activity) [17] to cycling: both works suggest that if designers want to facilitate togetherness in parallel exertion activities, they might benefit from considering supporting opportunities to affect each other's heart rate.

\section{Insight 2: Engaging the Interpretation of Heart Rate Data by Others can Facilitate Social Support}

In our study, we did not provide guidance on how to interpret the raw heart rate data (D3), yet the cyclists contextualized the data and added personalized information, attuned to their relationship, the environment and their partner's exertion state. Some of the cyclists said they enjoyed receiving feedback in this way, while all of them reported enjoying providing such feedback.

The Open Heart Helmet is basic in design, only displaying current heart rate. A more intelligent software system could interpret the heart rate data and provide the user with 
exercise statistics and instructions [3]. What such a system would have trouble offering, however, is the level of personalization that our participants provided to each other, powered by their empathy and understanding through sharing the same activity and context. A display on their handlebars (D2.2) could enable them to interpret their own data at any time (D1.1), making the system more efficient in terms of information transmission, satisfying those keen to access their heart rate data directly. Yet we encourage designers to also consider the qualities social involvement may bring to the user's experience. Our findings suggest that allowing others to interpret heart rate data can support a personalized delivery, which people may enjoy to receive and provide, facilitating social support. This personal form of sharing body data extends recent work [1] on using body data for real-time remote motivation and interaction through social media to co-located settings.

\section{Insight 3: Limited Access to Heart Rate Data is an Opportunity for Social Play}

Our cyclists expressed a desire to access their own heart rate, while at the same time recognized that it was this limited access that facilitated social play. We know from games research that limiting access to information can be a key game mechanic [23]. In the game Celebrity Heads, players must discover the name of the celebrity written on a card stuck to their forehead. Players can see the name cards of the other players, but not their own. Our cyclists were in a similar position, however, they were able to impact the heart rate displayed on their helmet (by cycling faster or slower, changing gears etc.), whereas the names on the cards in Celebrity Heads are constant. Our cyclists not only had access to their heart rate through their partner, but also through their own body (D4): they could generally sense when their heart rate increased or decreased.

There are more reasons why one might hide heart rate data. Stach et al. [25] hid absolute heart rate data (used as input for a video game) to balance players of different fitness levels while Dekker et al. [2] did so to indirectly and subtly influence the game experience without being obvious to the player. We extend this work by showing how limiting access to heart rate data can create an opportunity for social play. As such we also extend prior work on breathing interfaces, which suggests that different levels of access to breathing can be a powerful game mechanic [12].

\section{Insight 4: Location of Heart Rate Display Can Support Management of Distraction and Privacy Issues}

Participants cared for the management of potential distraction by heart rate displays, but were less concerned about privacy issues. Our system affected these aspects mainly through the location of the display: heart rate data was only available locally when riding behind the wearer of the display (D2), and only to those currently present (D1). Coincidental passersby might be able to glimpse at the cyclist's current heart rate, but none of the participants seemed concerned about this, we suggest this is because the moment is only temporary for a short time.
Our work extends proxemics' [8] notion that the location of interactive technology in relation to users and others matters in mobile exertion contexts. Our results suggest that the location of the display affects distraction issues and local privacy concerns (which is important as many exertion activities are performed outdoors, in public). Interestingly, Open Heart Helmet made heart rate data more public locally than for instance a mobile app, yet the potential for distraction for the wearer was lessened, as the rider behind (who might require less focus on the road or is a more experienced rider) could decide on suitable moments to convey the displayed information. While distraction opportunities might increase for the observer, we believe that designers of other wearable devices such as Google Glass and snowboarding goggles ${ }^{11}$ might benefit from considering making data more accessible to others who are in a better position to manage distraction issues.

\section{LIMITATIONS}

Our work offers insights into the design of interactive systems for cyclists, a relatively unexplored area [22]. We focused on particular aspects of cycling, therefore leaving others aside that can form part of future work. First, cycling sessions can last longer than in our study; still, we believe our work provides a first understanding on augmented social cycling experiences that can range from short rides to daylong activities. Second, we did not explore other mounting options for the display or sensed alternative body data. However, our dimensions provide designers with a range of options for exploring the design space. Third, we restricted our cyclists' interactions to a park without cars for safety reasons. Finally, study participants only used our system in a single session. More sessions may have revealed user's long-term strategies, yet the focus of our work was less on whether our design was successful in the long run, and more on the design insights that our study revealed. All considered, we believe we approximated an adequate "in the wild" experience, and hope our findings can serve as a valid starting point for more investigations around technology for social cycling experiences.

\section{CONCLUSION}

Open Heart Helmet is a system that displays heart rate data on a cycling helmet, designed to support a social exertion experience amongst pairs of cyclists. Our experience with cyclists using the system suggests that displaying heart rate on helmets is feasible and can support engaging experiences. Using body-worn displays in mobile exertion contexts is of interest because it facilitates interactions where the wearer often has less access to the information displayed than the people around him/her. Interviews with our cyclists reveal that such limitations can afford opportunities for engaging social exertion experiences. We identified a set of key dimensions and insights for designers to support the social and exertion aspect of an activity. By sharing our experiences with our helmet, we hope to expand

\footnotetext{
${ }^{11} \mathrm{http} / / \mathrm{www} . z e a l o p t i c s . c o m / \mathrm{z} 3 . \mathrm{html}$
} 
our understanding of the potential of heart rate data in mobile contexts to support social exertion experiences.

\section{ACKNOWLEDGEMENTS}

We thank Zephyr Technology Corporation, Bicycle Victoria Network, Alan Chatham, Cagdas 'Chad' Toprak, the Australian Research Council and all other supporters.

\section{REFERENCES}

1. Curmi, F., Ferrario, M.A., Southern, J. and Whittle, J., HeartLink: open broadcast of live biometric data to social networks. in Proc. CHI'13, (2013), ACM Press, 1749-1758.

2. Dekker, A. and Champion, E. Please biofeed the zombies: enhancing the gameplay and display of a horror game using biofeedback. Proc. DiGRA'07. (2007), 550-558.

3. Eyck, A., Geerlings, K., Karimova, D., Meerbeek, B., Wang, L., IJsselsteijn, W., De Kort, Y., Roersma, M. and Westerink, J. Effect of a virtual coach on athletes' motivation. in Persuasive Technology, Springer Berlin Heidelberg, 2006, 158-161.

4. Figl, K. and Gerhardt, T., Motivational and training effects of technical cycling equipment. in Proc. IADIS'08, (2008), 365-367.

5. Frederick-Recascino, C.M. and Schuster-Smith, H. Competition and intrinsic motivation in physical activity: A comparison of two groups. J. of Sport Behavior, 26 (3). (2003), 240-254.

6. Gerlach, A.L., Mourlane, D. and Rist, F. Public and private heart rate feedback in social phobia: A manipulation of anxiety visibility. Cognitive Behaviour Therapy, 33 (1). (2004), 36-45.

7. Gilleade, K., Dix, A. and Allanson, J., Affective videogames and modes of affective gaming: assist me, challenge me, emote me. in Proc. DiGRA'05, (2005), 547-554.

8. Greenberg, S., Marquardt, N., Ballendat, T., DiazMarino, R. and Wang, M. Proxemic interactions: the new ubicomp? interactions, 18 (1). (2011), 42-50.

9. Jones, E.M., Selker, T. and Chung, H., What you said about where you shook your head: a hands-free implementation of a location-based notification system. in Proc. CHI'07, (2007), ACM Press, 2477-2482.

10. LaChausse, R.G. Motives of competitive and noncompetitive cyclists. J. of Sport Behavior, 29 (4). (2006), 304-314.

11. Laukkanen, R.M.T. and Virtanen, P.K. Heart rate monitors: state of the art. J. of Sports Sciences, 16 (4). (1998), 3-7.

12. Marshall, J., Rowland, D., Rennick Egglestone, S., Benford, S., Walker, B. and McAuley, D., Breath control of amusement rides. in Proc. CHI'11, (2011), ACM Press, 73-82.

13. Mueller, F., Agamanolis, S. and Picard, R., Exertion interfaces: sports over a distance for social bonding and fun. in Proc. CHI'03, (2003), ACM Press, 561-568.
14. Mueller, F., Edge, D., Vetere, F., Gibbs, M.R., Agamanolis, S., Bongers, B. and Sheridan, J.G., Designing sports: a framework for exertion games. in Proc. CHI'11, (2011), ACM Press, 2651-2660.

15. Mueller, F., Gibbs, M.R. and Vetere, F., Design influence on social play in distributed exertion games. in Proc. CHI'09, (2009), ACM Press, 1539-1548.

16. Mueller, F., Vetere, F., Gibbs, M.R., Agamanolis, S. and Sheridan, J., Jogging over a distance: the influence of design in parallel exertion games. in Proc. SIGGRAPH'10, (2010), ACM Press, 63-68.

17. Mueller, F., Vetere, F., Gibbs, M.R., Edge, D., Agamanolis, S., Sheridan, J.G. and Heer, J., Balancing exertion experiences. in Proc. CHI'12, (2012), ACM Press, 1853-1862.

18. Nacke, L.E., Kalyn, M., Lough, C. and Mandryk, R.L., Biofeedback game design: using direct and indirect physiological control to enhance game interaction. in Proc. CHI'11, (2011), ACM Press, 103-112.

19. Nenonen, V., Lindblad, A., Häkkinen, V., Laitinen, T., Jouhtio, M. and Hämäläinen, P., Using heart rate to control an interactive game. in Proc. CHI'07, (2007), ACM Press, 853-856.

20. O'Hara, K., Glancy, M. and Robertshaw, S., Understanding collective play in an urban screen game. in Proc. CSCW'08, (2008), ACM Press, 67-76.

21. Page, M. and Vande Moere, A. Evaluating a wearable display jersey for augmenting team sports awareness. in Pervasive Computing, Springer, 2007, 91-108.

22. Rowland, D., Flintham, M., Oppermann, L., Marshall, J., Chamberlain, A., Koleva, B., Benford, S. and Perez, C., Ubikequitous computing: designing interactive experiences for cyclists. in Proc. MobileHCI'09, (2009), ACM Press, 1-11.

23. Salen, K. and Zimmerman, E. Rules of Play: Game Design Fundamentals. The MIT Press, 2003.

24. Schnädelbach, H., Egglestone, S.R., Reeves, S., Benford, S., Walker, B. and Wright, M. Performing thrill: designing telemetry systems and spectator interfaces for amusement rides Proc. $\mathrm{CHI}^{\prime} 08$, ACM Press, 2008, 1167-1176.

25. Stach, T., Graham, T., Yim, J. and Rhodes, R.E., Heart rate control of exercise video games. in Proc. Graphics Interface Conference'09, (2009), ACM Press, 125-132.

26. Weinberg, R.S. and Gould, D. Foundations of sport and exercise psychology. Human Kinetics, 2006.

27. Westerink, J., de Jager, M., van Herk, J., Bonants, R., de Kort, Y. and IJsselsteijn, W., Improving motivation in home fitnessing-A study into the effects of a virtual environment and a virtual coach giving biofeedback. in Proc. Philips User Interface Conference, (2003).

28. Wulf, V., Mueller, F., Moritz, S., Stevens, G. and Gibbs, M. Computer supported collaborative sports: an emerging paradigm. Digital Sport for Performance Enhancement and Competitive Evolution: Intelligent Gaming Technologies. (2009), Hershey, 136-149. 